\title{
FABRICACIÓN DE PUNTAS DE FIBRAS DE CARBONO PARA MICROSCOPÍA DE SONDA DE BARRIDO
}

\section{FABRICATION OF CARBON FIBER TIPS FOR ITS USE IN SCANNING PROBE MICROSCOPY}

\author{
Kevin Villegas ${ }^{1}$, Abel Gutarra ${ }^{2}$
}

RESUMEN

\begin{abstract}
Los microscopios de Fuerza Atómica (AFM) con sonda diapasón requieren puntas muy agudas y livianas para obtener imágenes topográficas de gran resolución. Por otro lado, los microscopios de Efecto Túnel (STM) necesitan puntas agudas y conductoras. Ambos requerimientos pueden ser obtenidos usando fibras de carbono como material base para la fabricación de las puntas, con la ventaja adicional de ser un material con bajo nivel de oxidación. En este trabajo se presentan algunos resultados iniciales de la técnica de disolución anódica que ha permitido obtener puntas de carbono de rango nanométrico. La celda electroquímica consistió en la fibra de carbono como ánodo ( $7 \mu \mathrm{m}$ de diámetro), un anillo de platino de $2 \mathrm{~mm}$ de diámetro como cátodo y $\mathrm{KOH}$ como electrolito. Una gota del electrolito se suspendió del anillo de platino para posteriormente introducir la fibra dentro de la gota. La polarización de la celda se obtuvo por medio de una interfase electroquímica en el modo de crono-amperómetro, el cual permitió registrar las curvas corriente-tiempo para diferentes voltajes escalón. Se encontraron correlaciones entre la forma final de la punta y la molaridad del electrolito, voltaje anódico, tiempo de disolución y profundidad de inmersión de la punta en el electrolito.

Por medio de microscopía electrónica de barrido se observó que se pueden obtener puntas con diámetros menores a $70 \mathrm{~nm}$ de manera reproducible.
\end{abstract}

Palabras clave.-Fibra de carbono, Punta nanométrica, Crono-amperometría.

\begin{abstract}
The Atomic Force Microscope (AFM) using a tuning fork as probe requires very sharp and light tips for obtaining topographic images with great resolution. On the other hand, Scanning Tunneling Microscopes (STM) needs sharp and conducting tips. Both requirements can be achieved using carbon fiber as the base material for the fabrication of the tips, with the additional advantage of being a material with a very low tendency to oxidize. In this work some initial results of the anodic dissolution technique are presented, which has permitted us to obtain the apex size of the tips in the nanometer scale. The electrochemical cell consists of the carbon fiber as the anode (7 $\mu$ m diameter), a $2 \mathrm{~mm}$ diameter Platinum ring as the cathode and Potassium Hydroxide as the electrolyte. A drop of the electrolyte is deposited on the Platinum ring and after that the carbon fiber is introduced into the drop. The polarization of the cell was obtained using a Potentiostat in Chrono-Amp mode, which permitted us to register Current vs. Time graphs for different values of potential applied. Correlations between the shape of the final tip and the molarity of the electrolyte, anodic potential, time for the anodic dissolution and the length of the carbon fiber beneath the airlelectrolyte interface were found. Using Scanning Electron Microscopy, it was observed that tips with diameter lower than $70 \mathrm{~nm}$ can be obtained in a reproducible way.
\end{abstract}

Keywords.-Carbonfibre, Nanometer tip apex size, Chrono-amp.

${ }^{1}$ Estudiante de Pregrado del Laboratorio de Materiales Nanoestructurados de la Facultad de Ciencias de la Universidad Nacional de Ingeniería, ${ }^{2}$ Dr. investigador de la Facultad de Ciencias, de la Universidad Nacional de Ingeniería.

TECNIA 22 (1) 2012 


\section{INTRODUCCIÓN}

Las técnicas de microscopía por efecto túnel (STM) [1] y de fuerza atómica (AFM) [2] son herramientas extremadamente útiles en la exploración de las propiedades de la materia en la nano escala. Ambas técnicas hacen uso de puntas finas para sondear las propiedades a escala atómica, con la resolución y la estabilidad que requieren diferentes tipos de materiales tanto orgánicos como inorgánicos.

Recientemente se están desarrollando microscopios mixtos que usan efecto túnel y fuerza atómica en forma combinada [3, 4]. Un caso particularmente interesante es la construcción de un AFM con sonda oscilante (diapasón) y que pueda operar como STM simultáneamente. Para ello se requiere de una punta muy liviana, para no reducir el factor de calidad del oscilador,pero que a la vez tenga conductividad eléctrica. Las fibras de carbono cumplen estos dos requisitos, y además son resistentes a la oxidación, que es frecuente en las puntas metálicas produciendo inestabilidad en la corriente túnel.

La disolución electroquímica es un método bastante conocido para fabricar puntas metálicas con algunos nanómetros de diámetro [5]. Sin embargo, la naturaleza de las reacciones químicas no son todavía muy claras cuando el electrodo es una fibra de carbono. La alta estabilidad del carbono y la estructura microscópica de la fibra hacen relativamente complejo modelar el proceso de disolución que lleva a la formación de la punta. En este trabajo se demuestra, que pese a esta dificultad, es posible obtener cierto grado de reproducibilidad en su fabricación.

\section{EXPERIMENTAL}

\section{Fijación de fibras sobre soporte conductor}

A diferencia de lo reportado en otros artículos donde sostienen las fibras individuales con un alambre conductor, [6,7], en este trabajo se comprimen varias fibras simultáneamente sobre láminas de cobre adhesivas. Para afirmar el contacto eléctrico, la lámina de cobre y las fibras se aseguran entre dos piezas rectangulares de acrílico por medio de tornillos. En la Fig.1se aprecian la forma y dimensiones de las fibras utilizadas. Son evidentes algunas fragmentos de suciedad adheridos a la superficie. En la Fig.2 se puede observar la forma irregular de la sección transversal de las fibras mostrando cavidades y rugosidades de varios cientos de nanómetros en promedio.

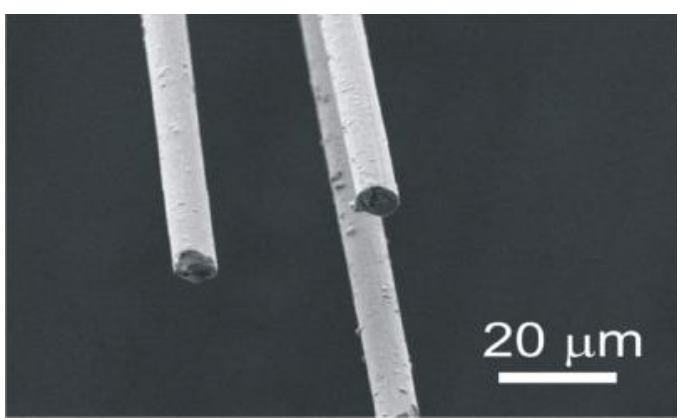

Fig. 1 Micrografía SEM de las fibras de carbono que se usa en el proceso electroquímico.

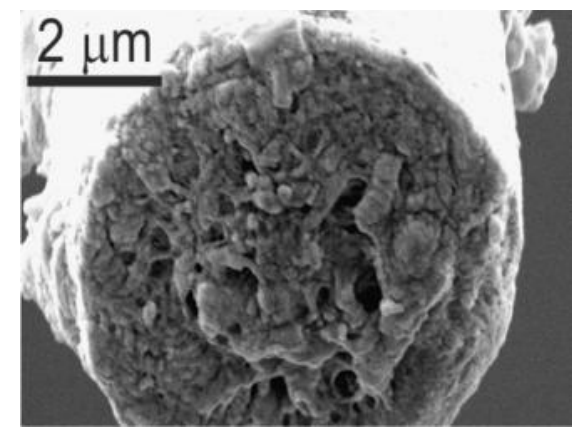

Fig. 2 Detallede la sección transversal de la fibra de carbono cuyo diámetro es $\sim$ de $7 \mu \mathrm{m}$.

\section{Posicionamiento de las fibras de carbono}

Por medio de posicionadores micrométricos, la pieza de acrílico se manipuló con facilidad hasta colocar alguna de las puntas en el centro de un anillo que actuó como cátodo. Ver Fig. 3.El anillo se desplazó verticalmente por medio de una mesa rígida de microscopio óptico que cuenta con un desplazador manual con una precisión de $\pm 1 \mu \mathrm{m}$.

\section{Celda electroquímica}

La celda electroquímica mostrada en Fig. 4 consiste en la fibra de carbono como ánodo, un anillo de platino fabricado con un alambre de 2,5 $\mathrm{mm}$ de diámetro y una solución electrolítica que, en forma de gota, llena el anillo de $2.0 \mathrm{~mm}$ de diámetro y se sostiene por tensión superficial. 
Para la polarización de los electrodos y el registro de datos, durante el proceso de disolución anódica, se utilizó un Potenciostato modelo 263 A, Princeton, controlado mediante un computador.

Adicionalmente se utilizó una micro cámara digital (Dino-Lote Pro con 40X de aumento) para verificar visualmente la aproximación de la fibra hacia el centro del anillo. Como electrolito, se usó Hidróxido de Potasio (KOH) en diferentes concentraciones dentro del rango de 1 a $16 \mathrm{M}$ y voltajes de polarización en un rango de $1.75 \mathrm{~V}$ a $3.5 \mathrm{~V}$

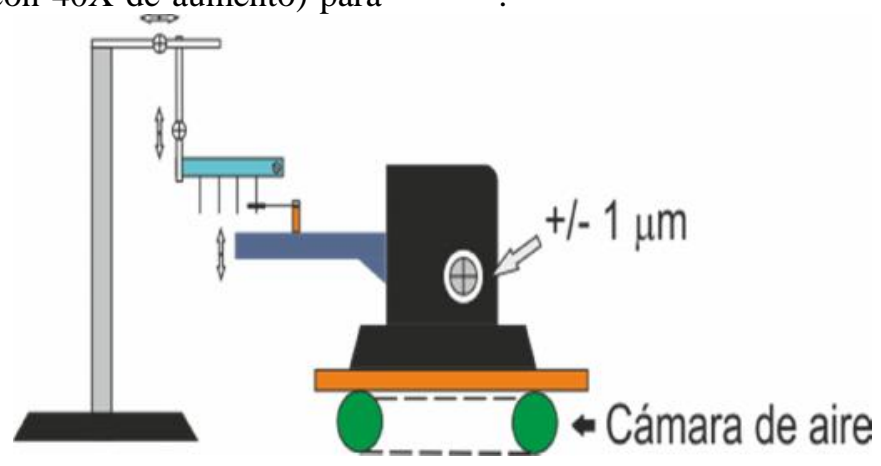

Fig. 3 Sistema de posicionamiento de las fibras de carbono.

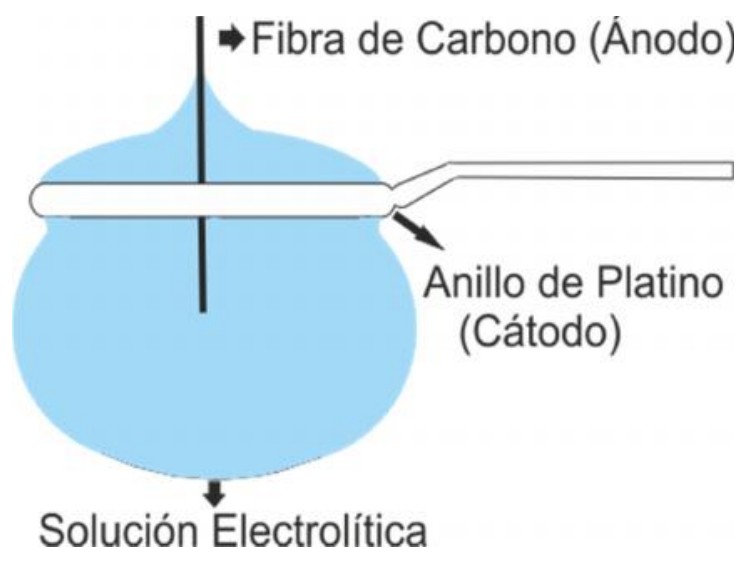

Fig. 4 Celda electrolítica, donde se realiza la disolución anódica.

El proceso comienza cuando se le aplica el voltaje anódico a la fibra y se produce el adelgazamiento de la zona de la fibra que se encuentra en la interfase aire-electrolito.

La corriente decrece hasta hacerse nula en el momento en que la parte sumergida de la fibra se separa de la parte superior.

Un gráfico típico corriente-tiempo durante del proceso se muestra en la Fig. 5.

\section{RESULTADOS Y DISCUSIÓN}

\section{Disolución anódica con variaciones de voltaje}

Se realizaron barridos lineales de voltaje dentro del rango en que se produce la disolución de la fibra, con velocidades de $1 \mathrm{~V} \mathrm{~min}^{-1}$ y $2 \mathrm{~V} \mathrm{~min}$.

Las curvas corriente-voltaje generados en estas condiciones se muestran en la Fig. 6. Se puede apreciar que aproximadamente en $2.3 \mathrm{~V}$ y $2.8 \mathrm{~V}$ se producen incrementos de corriente asociados a la disolución de la fibra. 


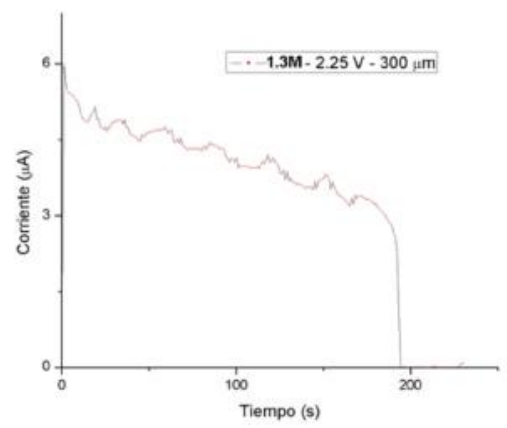

Fig. 5 Gráfica corriente vs tiempo de una disolución anódica común.
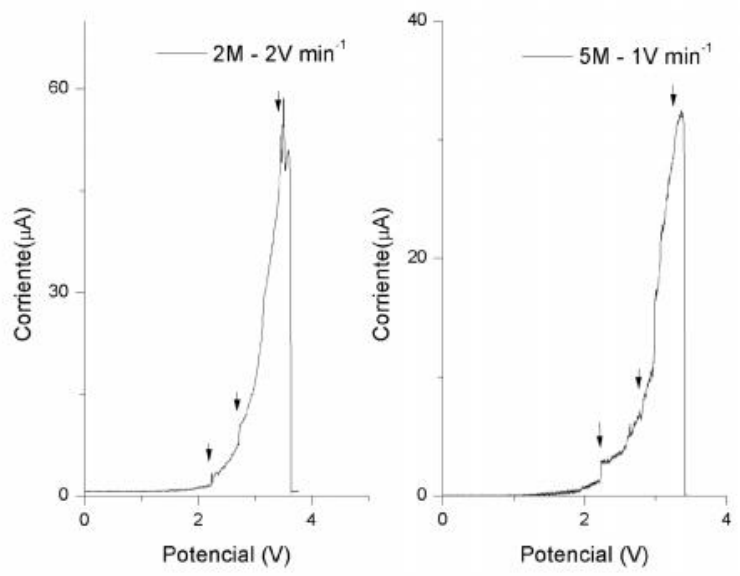

Fig.6 Curvas corriente-voltaje para diferentes molaridades $(2 \mathrm{M}, 5 \mathrm{M} \mathrm{KOH})$ y diferentes velocidades de barrido $\left(1 \mathrm{~V} \mathrm{~min}^{-1}, 2 \mathrm{~V} \mathrm{~min}^{-1}\right)$. Las flechas indican los cambios bruscos de corriente.

Los resultados anteriores sugieren procesos de transferencia de carga bien definidos para ciertos voltajes. Para poder verificarlo, se hizo una prueba de voltametría cíclica a mayor velocidad de barrido en el rango de voltajes en que se produce la disolución de la fibra. El resultado de esta prueba se muestra en la Fig. 7. Se observan con mayor detalle los tres incrementos de corriente y dos mesetas en valores de voltaje cercanos a los encontrados en la Fig. 6.

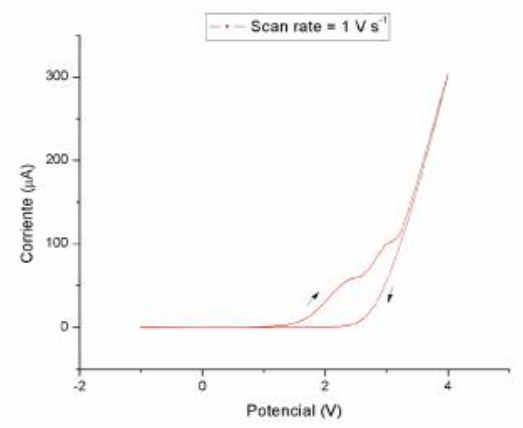

Fig. 7 Voltametría cíclica que pone en evidencia procesos de transferencia de carga en voltajes definidos. 


\section{Efecto del voltaje anódico}

Para la fabricación de las puntas se fijan el voltaje de polarización, la molaridad del electrolito y la profundidad de inmersión de la punta. Un primer grupo de pruebas se realizaron variando el voltaje anódico, fijando las demás variables. El monitoreo de la forma de las puntas obtenidas se hizo con microscopía óptica, tal como se puede ver en la Fig. 8. La punta se va agudizando a medida que el voltaje aumenta. Destaca la agudeza de la punta obtenida a $2,25 \mathrm{~V}$.

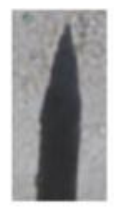

$2.75 \mathrm{~V}$

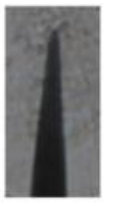

$2.5 \mathrm{~V}$

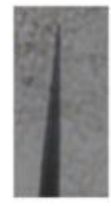

$2.25 \mathrm{~V}$

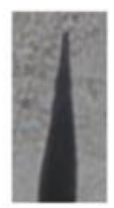

$2.0 \mathrm{~V}$

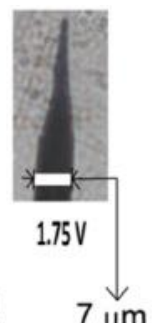

$7 \mu \mathrm{m}$

Fig. 8 Micrografías de una serie de puntas obtenidas a diferentes voltajes anódicos.

\section{Efecto de la molaridad}

Manteniendo el voltaje anódico en $2 \mathrm{~V}$, se analizó el efecto de la concentración del electrolito sobre la forma y dimensiones de la punta. Las micrografías ópticas obtenidas se muestran el Fig. 9. El efecto de la molaridad durante el ataque electroquímico no reside en el tamaño del radio de curvatura de la parte final de la punta, sino más bien, en su forma.
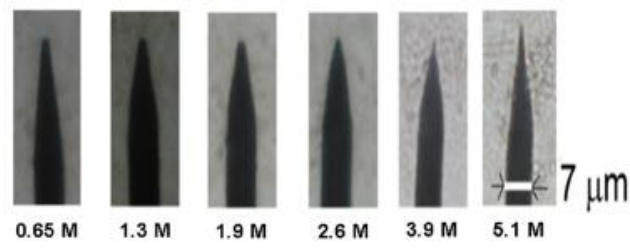

Voltaje aplicado $=2.5 \mathrm{~V}$

Fig. 9 Micrografías de una serie de puntas obtenidas a diferentes concentraciones de KOH.

Además del voltaje anódico y la concentración del electrolito, es posible variar la geometría de la punta controlando la profundidad de inmersión de la fibra dentro del electrolito. En las Figuras 10 y 11 se pueden apreciar dos puntas con perfiles correspondientes a cuellos de alta curvatura y baja curvatura respectivamente, con radios en el extremo de la punta de 50 y $33 \mathrm{~nm}$.

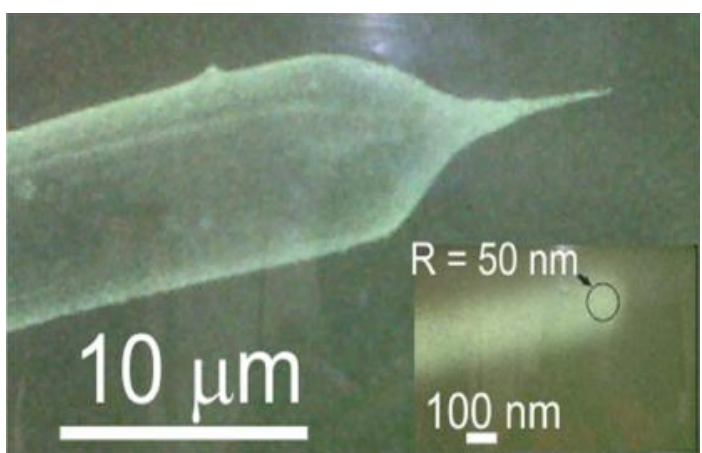

Fig. 10Punta con cuello de alta curvatura. 


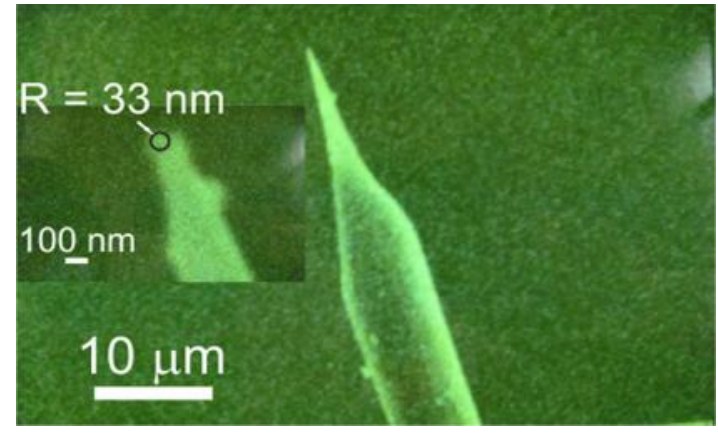

Fig. 11Punta con cuello de baja curvatura.

\section{CONCLUSIONES}

A partir de fibras de carbono comerciales se han obtenido puntas con radios de hasta $33 \mathrm{~nm}$. El procedimiento utilizado es simple de implementar y permite un buen grado de reproducibilidad.

Las reacciones químicas específicas que determinan la formación de la punta, aún son materia de investigación, pero las técnicas electroquímicas como la cronoamperometría y la voltametría cíclica utilizadas en este trabajo ponen en evidencia reacciones de transferencia de carga bien definidos.

\section{AGRADECIMIENTOS}

A J. Morán y J. Cousty por proporcionarnos las fibras de carbono para el trabajo y por fructíferas discusiones; V. Quinde por su ayuda técnica; $\mathrm{H}$. Alarcón por facilitarnos la interfase electroquímica;
S. Petrick por su ayuda con las fotografías ópticas;

C. Luyo y M. Fischer por su apoyo con el SEM.

\section{REFERENCIAS}

1. Binning, G., Rohrer H., "Helv.Phys". Acta55 726-35, (1982).

2. Binning G, Quate $\mathbf{C} \mathbf{F}$ and Gerber $\mathbf{C}$. "Phys. Rev". Lett.56 930-3, (1986).

3. Edwards, H., Taylor L., Duncan, W.,Melmed, A. J.,“J. Appl. Phys.” 82 980-4, (1997).

4. Giessibl, F.,“J. Appl. Phys”. Lett.73 3986-8, (1998).

5. W. J. McG. Tegart., "The Electrolytic and Chemical" Polishing of Metals in Research and Industry, 1 sted.Pergamon, New York, (1956).

6. Castellanos-Gomez, A., Agraït N, RubioBollinger,G.,

"Nanotechnology;21(14):145702, (2010).

7. Sripirom J., Noor, S.,Köhler, U., Schulte. A., Carbon, 49:2402-2412 (2011).

8. Allen, J., Bard, L. R.,"Faulkner ElectrochemicalMethods: fundamental and applications" $-2^{\text {nd }}$ ed. John Wiley \& Sons, Inc. 2000.

Correspondencia: k.villegas33@gmail.com 